\title{
DESEMPENHO DA IRRIGAÇÃO E FERTIRRIGAÇÃO AVALIADAS POR CONTROLE ESTATÍSTICO DE QUALIDADE
}

\author{
André Luiz Justi ${ }^{1}$, Paula Mayumi Saizaki ${ }^{2}$
}

\section{RESUMO}

A avaliação de um sistema de irrigação pode ser feita com uso da uniformidade de distribuição de água, sendo o Coeficiente de Uniformidade de Christiansen (CUC) o mais utilizado, pois além de ser uma fonte de avaliação de seu bom funcionamento, também é uma forma de verificar sua viabilidade de implantação e operação. Com este trabalho objetivou-se utilizar o Controle Estatístico de Qualidade (CEP), por meio do índice de capacidade do processo, para avaliar a uniformidade de distribuição de água de um sistema de irrigação por aspersão convencional operando com água e realizando a fertirrigação. Os ensaios foram conduzidos no Núcleo Experimental de Engenharia Agrícola NEEA - pertencente a Universidade Estadual do Oeste do Paraná - UNIOESTE - Campus Cascavel - Paraná, com o uso de 2 aspersores modelo Super10, marca NAANDAN, espaçados $9 \mathrm{~m}$ entre si e 18 coletores com espaçamento de $3 \mathrm{~m}$. Os fatores climáticos foram monitorados e coletados em intervalos de 10 min por uma estação climatológica sem fio, marca La Crosse ao longo de 25 ensaios de 1 h para irrigação e fertirrigação. Para os ensaios de irrigação, foi encontrado um CUC médio de 79,72\% sendo que o valor médio de velocidade do vento foi de $1,85 \mathrm{~m} \mathrm{~s}^{-1} \mathrm{e} \mathrm{com}^{-1}$ fertirrigação o CUC encontrado foi de $77,14 \%$ com velocidade média do vento de $1,50 \mathrm{~m} \mathrm{~s}^{-1}$. Foram calculados os limites inferior e superior de controle para elaboração do gráfico de controle de qualidade bem como o índice de capacidade do processo para os coeficientes de uniformidade.Os resultados obtidos permitiram concluir que o controle estatístico de qualidade é uma ferramenta de grande auxílio para classificarsistemas de irrigação na busca da otimização do uso da água.

Palavras-Chave: Controle de qualidade, nitrogênio, aspersores.

\section{ABSTRACT \\ IRRIGATION AND FERTIGATION PERFORMANCE EVALUATED BY STATISTICAL QUALITY CONTROL}

The evaluation of an irrigation system can be made with use of uniformity of water distribution, whereas the Christiansen's Coefficient of Uniformity (CCU) is the most used, since it is a source of evaluation of its proper functioning as well as a way to check its viability deployment and operation. This work has the objective to use the Statistical Quality Control (SQC), through the process capability index to evaluate the uniformity of water distribution from a conventional sprinkler irrigation system operating with water and performing fertigation. The tests were conducted at the Experimental Center of Agricultural Engineering - NEEA - belonging to the State University of West of Paraná - UNIOESTE - Campus Cascavel - Paraná, using 2 sprinklers Super10 model (NAANDAN), spaced 9 m apart and 18 collectors with a distance of $3 \mathrm{~m}$. Climatic factors were monitored and collected at 10 min intervals by a wireless meteorological station (La Crosse) over 25 trials of $1 \mathrm{~h}$ each for irrigation and fertigation. For irrigation trials, an average CCU of $79.72 \%$ was found and the wind average speed was $1.85 \mathrm{~m} \mathrm{~s}^{-1}$ and for fertigation, the CCU was $77.14 \%$ with an average wind speed of $1.50 \mathrm{~m} \mathrm{~s}^{-1}$. The upper and lower control limits were calculated for the quality control charts and process capability index for the uniformity coefficients. Results showed that the Statistical Quality Control is a powerful auxiliary tool forclassifying irrigation systems in the pursuit of optimization of water use.

Keywords: Quality control, nitrogen, sprinklers.

\section{Recebido para publicação em 09/09/2014. Aprovado em 16/10/2015.}

1 - Engenheiro Agrícola, Professor Adjunto UFPR, Jandaia do Sul-PR, e-mail: aljusti@ufpr.br

2 - Engenheira de Bioprocessos e Biotecnologista, Professora Assistente UTFPR, Apucarana-PR, e-mail: paula.saizaki@gmail.com 


\section{INTRODUÇÃO}

Incrementar a produção de alimentos nos dias de hoje é uma necessidade para a agricultura e a irrigação tem papel fundamental nesse incremento, tanto pelo aumento da produção como pela possibilidade de cultivo em áreas onde o clima não é favorável.

Pode-se definir a irrigação, de acordo com Frizzone et al. (2005) e Mantovani et al. (2009), como a aplicação racional de água, sob forma de chuva artificial ao solo, em intervalos definidos e em quantidade suficiente para fornecer às espécies vegetais umidade ideal para seu pleno desenvolvimento com mínimo impacto ambiental.

De acordo com Mateos (1998), a distribuição da água aplicada dos sistemas de aspersão é um processo em duas etapas: uma onde ocorre aplicação da água na superfície do solo ou da cultura e redistribuição da água aplicada no interior do solo. Apesar dos efeitos positivos da redistribuição da água no solo, em alguns casos, pode anular os efeitos da desuniformidade de aplicação (LI; RAO, 2003). Frizzone et al. (2007), enfatiza que devese considerar que a uniformidade do teor de água do solo e a produtividade das culturas irrigadas são bastante dependentes da uniformidade com que a água é aplicada durante a primeira fase desse processo.

Para que os sistemas de irrigação possam desempenhar tal papel, faz-se necessários índices de uniformidade altos, pois a uniformidade de distribuição de sistemas de irrigação é de suma importância para a otimização do uso da água, com repercussões diretas na eficiência e na produção (CARRIÓN et al., 2001) e, geralmente, a uniformidade de distribuição é a principal maneira usada para determinar seum sistema de irrigação é aceitável ou não (BRENNAN, 2008).

Existem vários meios de se obter a uniformidade de distribuição de sistemas de irrigação, sendo que o mais difundido e utilizado é o Coeficiente de Uniformidade de Christiansen (CUC) e, em campo, os fatores que afetam a uniformidade vão desde falhas de projeto à velocidade do vento, e em termos de fertirrigação, inclui-se ainda o entupimento dos emissores.

Em busca desse crescimento, usa-se a aplicação de fertilizantes junto à irrigação, que para Klar
(2000), aplicar fertilizantes via água de irrigação é uma técnica que tomou grande impulso, dada adequabilidade dos adubos líquidos para as plantas.

Aplicar fertilizantes via irrigação exige controle dos fatores que possam afetar a uniformidade de distribuição de água do mesmo, pois de acordo com Carrión et al. (2001) a mesma possui um papel importante na otimização do uso da água, com repercussões diretas na eficiência e na produção.

Em campo, a importância de uniformidade de irrigação e sua influência no rendimento e eficiência de aplicação é bem visível. São usados frequentemente vários parâmetros de desempenho para descrever a distribuição de água de irrigação no campo, sendo que um dos mais importantes é a uniformidade de distribuição (CLEMMENS; MOLDEN, 2007); dentre os coeficientes de uniformidade, o mais utilizado é o Coeficiente de Uniformidade de Christiansen (CUC).

Controle estatístico de qualidade se refere a um conjunto de métodos amplamente utilizadospara monitorar e melhorar a produtividade em processos e sistemas produtivos (STOUMBOS et al., 2000), com suas bases desenvolvidas pelo estatístico Shewhart, do Bell Laboratories, no final da década de 1920, sendo divulgada mundialmente por Deming, em 1953 (VEIGA et al., 2015).

Utilizar apenas uma medida para avaliar um sistema de irrigação restringe-se apenas a uma situação localizada; no caso do CUC, a apenas um ensaio, tanto de irrigação como de fertirrigação. Em situações similares, segundo Montgomery (2009) técnicas de engenharia de qualidade tornam-se ferramentas que podem realizar avaliações ao longo de um período estabelecido, verificando a variabilidade em função do tempo, sendo geralmente utilizadas quando o processo se encontra estável, ou seja, quando o processo se encontra sob controle estatístico e quando a variável de interesse apresenta distribuição próxima da distribuição normal. Assim, conforme Justi et al. (2010), o índice de capacidade do processo quando usado para avaliação de sistemas de irrigação tem comportamento diretamente proporcional entre o aumento da uniformidade de distribuição e o índice de capacidade do processo.

Em vista disso, buscou-se com este trabalho avaliar um sistema de irrigação por aspersão convencional através da realizaçãoda irrigação e da fertirrigação e, então, comparar seus desempenhos

\section{REVENG} 541-551p. 
com base nas técnicas de controle de qualidade e índice de capacidade do processo.

\section{MATERIAL E MÉTODOS}

O experimento foi conduzido no Núcleo Experimental de Engenharia Agrícola, NEEA, o qual faz pertence à Universidade Estadual do Oeste do Paraná - UNIOESTE, com uma altitude média de $750 \mathrm{~m}$, clima subtropical temperado úmido de acordo com a classificação climática de Köppen, a precipitação média anual é de $1971 \mathrm{~mm}$ e a temperatura média anual é de $19,6{ }^{\circ} \mathrm{C}$.

$\mathrm{O}$ sistema de irrigação avaliado constituiu-se de uma lateral na qual foram instalados dois aspersores da marca $N A A N D A N$, série categoria Irristand, modelo Super10, bocal azul, não compensante, e, segundo catálogo fornecido pelo fabricante, operando com uma pressão de serviço de $300 \mathrm{kPa}$, sua vazão é de $395 \mathrm{~L} \mathrm{~h}^{-1}$, com um diâmetro irrigado de $16 \mathrm{~m}$. Ainda segundo o fabricante, utilizando o espaçamento de $9 \mathrm{~m} \times 9 \mathrm{~m}$, a uniformidade de aplicação desse emissor pode chegar a 92\%.

$\mathrm{O}$ sistema foi instalado usando um adaptador $\mathrm{F}$ de $1 / 2$ " fixado em uma haste galvanizada de $8 \mathrm{~mm}$ diâmetro por $1,2 \mathrm{~m}$ de altura, ligado a lateral por um tubo de Polietileno, com diâmetros externo e interno de 13 e $12 \mathrm{~mm}$ respectivamente.

A lateral foi montada com tubos de Polietileno de $25 \mathrm{~mm}$ de diâmetro e a linha principal foi instalada com tubos de Policloreto de Vinila (PVC) com $50 \mathrm{~m}$ de comprimento, ligada a um sistema de bombeamento composto por três bombas marca THEBE, modelo M2B-12X, com potência máxima de $0,5 \mathrm{cv}$, operando a $3500 \mathrm{rpm}$, para uma vazão máxima de $5,1 \mathrm{~m}^{3} \mathrm{~h}^{-1} \mathrm{e}$ uma pressão máxima de $260 \mathrm{kPa}$.

Em função da instalação dos registros para ramificar a saída de água da bomba, ocorreu uma perda de carga de $100 \mathrm{kPa}$ comparando-se com os ensaios que utilizaram apenas água.

A pressão foi monitorada por meio de um manômetro e foram tomadas as precauções para a sua variação não ultrapassar os $4 \%$ admitidos pela NBR 7749 (ABNT, 1999) para ensaios de aspersores. Durante os ensaios, a pressão utilizada para a irrigação foi de $300 \mathrm{kPa}$ e para fertirrigação em $200 \mathrm{kPa}$, dadas perdas de carga em função da instalação do sistema de injeção de fertilizante.

O fertilizante utilizado foi o nitrogênio, sob a forma de ureia na concentração de $45 \%$ de nitrogênio para cada quilo, tendo um total de $10 \mathrm{~kg}$ de ureia aplicado, somando 4,5 kg de nitrogênio.

$\mathrm{O}$ fertilizante foi armazenado em um reservatório com $0,63 \mathrm{~m}$ de diâmetro e $0,90 \mathrm{~m}$ de altura, com volume total de 280 litros. Foi utilizado um injetor do tipo Venturi para realizar a fertirrigação.

Os dados climáticos foram monitorados ao longo de cada ensaio, com intervalo de 10 minutos, utilizando uma estação climatológica sem fio (Wireless Weather Station) modelo WS-2310 da La Crosse Technology, observando velocidade do vento, temperatura e umidade relativa do ar.

Ao todo foram realizadas 25 irrigações utilizando apenas água e 25 fertirrigações, seguindo a quantidade de amostras indica pro MONTGOMERY (2009) para aplicação dos testes de controle de qualidade, tendo todos os ensaios duração de $1 \mathrm{~h}$ e características de operação semelhantes.

Para avaliar o sistema, além da uniformidade de distribuição, que no experimento foi o Coeficiente de Uniformidade de Christiansen (CUC), dado pela Equação 1, neste experimento seguiu-se a metodologia sugerida por WERKEMA (1995), realizando-se a avaliação da capacidade do processo, em que se calculou o índice de capacidade (Cp). Comumente oíndice $\mathrm{Cp}$ é utilizado quando o processo se encontra estatisticamente estável; $\mathrm{C}_{\mathrm{p}}$ é definido pela Equação 2.

$C U C=100\left(1-\frac{\sum_{i=1}^{n}\left|X_{i}-Z\right|}{n \tilde{R}}\right)$

em que,

CUC - Coeficiente de Uniformidade de Christiansen, \%;

$X_{i}$ - Precipitação no coletor de ordem i, mm;

$\bar{X}$ - Média aritmética das precipitações, mm; e $\mathrm{n}$ - Número de coletores;

$C_{p}=\frac{L S E-L I E}{6 \sigma}$

em que,

- LIE e LSE são os limites inferior e superior de especificação, respectivamente.

$\sigma$ - desvio padrão populacional da variável em estudo. 
O CPL (capacidade do processopelo limite inferior) é dado pela Equação 3.

$$
C_{P L}=\frac{\mu-L I E}{3 \sigma}
$$

Os limites inferior e superior de especificação são dados pelas equações $4 \mathrm{e}$ 5 , respectivamente.

$$
L I E=\mu-3 \frac{\sigma}{\sqrt{n}}
$$

$$
L S E=\mu+3 \frac{\sigma}{\sqrt{n}}
$$

em que,

$\mu=$ média populacional;

$n=$ número de amostras; $\mathrm{e}$

${ }_{\sigma^{\sigma}}{ }^{\sigma}=$ desvio padrão populacional.

Segundo WERKEMA (1995), a classificação do processo se faz da seguinte forma: se o valor de $\mathrm{Cp} \geq 1,33$, o processo é capaz ou adequado segundo as especificações; se $1 \leq \mathrm{Cp}<1,33$, o processo é aceitável; se $\mathrm{Cp}<1$, o processo é incapaz ou inadequado. A definição de $\mathrm{Cp}$ assume implicitamente que o processo está centrado no valor nominal da especificação. Se o processo não estiver centrado, sua capacidade real será menor do que a indicada por $\mathrm{Cp}$.

Os testes de controle de qualidade foram realizados calculando o índice de capacidade do processo (Equação 2). Para irrigação foi fixado como valor alvo, o informado pelo fabricante, que para a pressão de serviço de $300 \mathrm{kPa}$ a lâmina média aplicada é $4,9 \mathrm{~mm} \mathrm{~h}^{-1}$ e como limite inferior, o valor de lâmina do ensaio cujo CUC foi $80,05 \%$, por ser o mais próximo do mínimo aceitável pela norma, fixando assim a lâmina de $2,82 \mathrm{~mm} \mathrm{~h}^{-1}$. Para fertirrigação, foi utilizado o valor de lâmina média de $3,9 \mathrm{~mm} \mathrm{~h}^{-1}$, e o limite inferior dado pelo ensaio com CUC mais próximo do mínimo aceitável pela norma, ou seja, 2,056 $\mathrm{mm} \mathrm{h}^{-1}$. Para calcular o Limite Inferior de Especificação ou Controle (LIE) foi utilizada a Equação 4.

\section{RESULTADOS E DISCUSSÃO}

Os Quadros 1 e 2 apresentam a estatística descritiva para os ensaios com irrigação e fertirrigação, respectivamente. Foram calculados a média, desvio padrão, coeficiente de variação, valores de máximo e mínimo para o CUC, a velocidade do vento, umidade relativa do ar e temperatura para ambos os ensaios.

Foi realizado o teste de normalidade de Anderson-Darling em nível de 5\% de significância, onde todas as variáveis apresentaram distribuição normal para o sistema operando apenas a irrigação. Ao aplicar o mesmo teste para o sistema trabalhando a fertirrigação, a variável CUC não apresentou distribuição normal.

O CUC médio das 25 irrigações foi $79,72 \%$, e a velocidade do vento média, $1,85 \mathrm{~m} \mathrm{~s}^{-1}$. Os valores máximos de CUC e velocidade do vento foram $89,45 \%$ e $2,97 \mathrm{~m} \mathrm{~s}^{-1}$, respectivamente. Os valores de desvio-padrão obtidos a partir dos dados foram $3,74 \%$ e $0,70 \mathrm{~m} \mathrm{~s}^{-1}$ para CUC e velocidade do vento, respectivamente. $\mathrm{O}$ coeficiente de variação para o CUC foi $4,72 \%$ e para a velocidade do vento $37,98 \%$. Quanto aos fatores climáticos, foram encontrados valores entre 30 e $58 \%$ para umidade relativa e 27,30 e $41,80{ }^{\circ} \mathrm{C}$ para a temperatura, resultando nos valores médios de $46,04 \%$ e 32,59 ${ }^{\circ} \mathrm{C}$.

Para os ensaios com o sistema realizando a fertirrigação, o valor médio encontrado de CUC para as 25 fertirrigações foi $77,14 \%$ e a velocidade do vento médio, $1,50 \mathrm{~m} \mathrm{~s}^{-1}$. Ao longo do experimento os valores máximos de CUC e velocidade do vento foram de $81,04 \%$ e $2,18 \mathrm{~m} \mathrm{~s}^{-1}$, respectivamente. Os valores de desvio padrão obtidos a partir dos dados foram $2,79 \%$ e $0,31 \mathrm{~m} \mathrm{~s}^{-1}$ para CUC e velocidade do vento, respectivamente.Quanto ao coeficiente de variação, para o CUC o mesmo foi 3,62\% e para a velocidade do vento, 20,84\%. Quanto aos fatores umidade relativa e temperatura, foram encontrados valores entre 71 e $41 \%$ para umidade relativa e 21,1 e $35,9^{\circ} \mathrm{C}$, e valores médios de $54,24 \%$ e 30,7 ${ }^{\circ} \mathrm{C}$.

FARIA et al. (2009), indica em seu estudo de simulação da uniformidade da irrigação de sistemas convencionais de aspersão operando sob diferentes condições de vento, quando o autor cita que a análise do desempenho do modelo ajustado não pode deixar de considerar que as diferenças nas estimativas de CUC encontradas não refletem

\section{REVENG}

541-551p. ENGENHARIA NA AGRICULTURA, VIÇOSA - MG, V.23 N.6, NOVEMBRO / DEZEMBRO 2015 
Quadro 1. Estatística descritiva dos resultados obtidos para o CUC da irrigação, velocidade do vento, umidade relativa do ar e temperatura para a irrigação.

\begin{tabular}{crrrr}
\hline Análise & $\begin{array}{c}\text { CUC } \\
(\%)\end{array}$ & $\begin{array}{c}\text { Velocidade do } \\
\text { vento } \\
\left(\mathbf{m} . \mathbf{s}^{-1}\right)\end{array}$ & $\begin{array}{c}\text { Umidade } \\
\text { Relativa } \\
(\%)\end{array}$ & $\begin{array}{c}\text { Temperatura } \\
\left({ }^{\circ} \mathbf{C}\right)\end{array}$ \\
\hline Norma para o CUC & 80 & -- & -- & -- \\
Média & 79,72 & 1,85 & 46,04 & 32,59 \\
Desvio Padrão & 3,74 & 0,70 & 8,19 & 3,94 \\
Variância & 14,05 & 0,49 & 67,12 & 15,56 \\
Coeficiente & & & & \\
De Variação & 4,72 & 37,98 & 17,80 & 12,10 \\
Mínimo & 72,25 & 0,44 & 30,00 & 27.30 \\
Máximo & 89,45 & 2,97 & 58,00 & 41,80 \\
Teste de Normalidade & & & & Não se \\
(Anderson -Darling) & Não se & Não se & rejeita* & rejeita* \\
\hline
\end{tabular}

*Nível de 5\% de significância

Quadro 2. Estatística descritiva dos resultados obtidos para o CUC da fertirrigação, velocidade do vento, umidade relativa do ar e temperatura.

\begin{tabular}{|c|c|c|c|c|}
\hline Análise & $\begin{array}{r}\text { CUC } \\
(\%)\end{array}$ & $\begin{array}{c}\text { Velocidade do } \\
\text { vento } \\
\left(\mathrm{m} . \mathrm{s}^{-1}\right) \\
\end{array}$ & $\begin{array}{c}\text { Umidade } \\
\text { Relativa } \\
(\%) \\
\end{array}$ & $\begin{array}{c}\text { Temperatura } \\
\left({ }^{\circ} \mathbf{C}\right)\end{array}$ \\
\hline Norma para o CUC & 80 & \multirow{2}{*}{1,50} & -- & \multirow{2}{*}{30,70} \\
\hline Média & 77,14 & & 54,24 & \\
\hline Desvio Padrão & 2,79 & \multirow{2}{*}{0,09} & 7,13 & 3,01 \\
\hline Variância & 7,81 & & 50,77 & 9,08 \\
\hline Coeficiente & & & & \\
\hline De Variação & 3,62 & \multirow{3}{*}{$\begin{array}{r}20,84 \\
0,86 \\
2,18\end{array}$} & 13,14 & \multirow{3}{*}{$\begin{array}{l}9,82 \\
21,1 \\
35,9\end{array}$} \\
\hline Mínimo & 70,91 & & 41,00 & \\
\hline Máximo & 81,04 & & 71,00 & \\
\hline $\begin{array}{l}\text { Teste de Normalidade } \\
\text { (Anderson-Darling) }\end{array}$ & Rejeita-se* & $\begin{array}{l}\text { Não se } \\
\text { rejeita* }\end{array}$ & Não se rejeita* & $\begin{array}{l}\text { Não se } \\
\text { rejeita* }\end{array}$ \\
\hline
\end{tabular}

* A nível de $5 \%$ de significância.

apenas as deficiências do modelo ajustado, mas também refletem a dificuldade de se obter, ao longo do tempo o mesmo grau de estabilidade nos valores dos parâmetros climáticos considerados nas simulações, dadas dificuldades operacionais encontradas em relação aos fatores climáticos, o que corrobora com o observado neste estudo, sendo o comportamento dos fatores climáticos, em especial do vento, é um item de difícil controle e manutenção de valores estáveis, afetando 
diretamente os valores obtidos de CUC.

LI et al. (2007), estudando injeção de fertilizante com injetor Venturi, verificaram que para um determinado tanque com uma pressão diferencial que liberou fertilizante em uma taxa que decresceu com o tempo, ocorreu uma variação consideravelmente maior que a da aplicação de água, confirmando os resultados obtidos neste trabalho, em que se registrou uma diferença de pressão entre a aplicação de água e a fertirrigação, que somada à variação da velocidade do vento, influenciou nos resultados.

A diferença de uniformidade de aplicação entre uso de água e fertirrigação encontrada neste estudo confirma a afirmação de LI et al. (2007), onde a relação entre uniformidade de aplicação de água e uniformidade da fertirrigação para um sistema de irrigação está fortemente associada com método de injeção, pois um sistema que produz uma aplicação de água uniforme não significa, necessariamente, que haverá uma distribuição uniforme na fertirrigação.

Soares; Nascimento (1998), avaliando aspersores em um perímetro irrigado, citam terem encontrado CUC de $79 \%$, utilizando pressão de $240 \mathrm{kPa}$, ou seja, uma pressão próxima da utilizada neste experimento, com resultados semelhantes aos obtidos para a irrigação aplicando água.
Em relação a pressão de serviço, Solomon (2008) justifica o uso de uma pressão baixa afirmando que há uma tendência em se diminuir a pressão de serviço em função do alto custo de energia. Analisando o funcionamento do sistema estudado, mesmo tendo trabalhado com uma pressão de $200 \mathrm{kPa}$, os valores de uniformidade oscilaram entre 70 e $81 \%$, indicando a presença de outro fator de influência, a variação do vento, que na região onde o sistema foi instalado é considerada um fator importante quando do uso da irrigação por aspersão.

Na Figura 1, pode-se verificar a correlação entre o CUC da fertirrigação e a velocidade do vento. O coeficiente de determinação $\left(\mathrm{R}^{2}\right)$ foi de $68,4 \%$, confirmando o que Burt et al. (1997) afirmaram, que não somente o vento influencia no CUC, mas também as diferenças de pressão, orientação vertical do aspersor, duração da irrigação, além do próprio design do emissor.

Já na Figura 2, verifica-se uma elevada correlação entre o CUC e a variação da velocidade do vento ao longo dos ensaios, com coeficiente de determinação $\left(\mathrm{R}^{2}\right)$ de $83,6 \%$.

Esses resultados assemelham-se aos de Souza et al. (2008), que estudando o desempenho de um sistema de irrigação por aspersão convencional em vila rural, devido a fatores climáticos, obtiveram

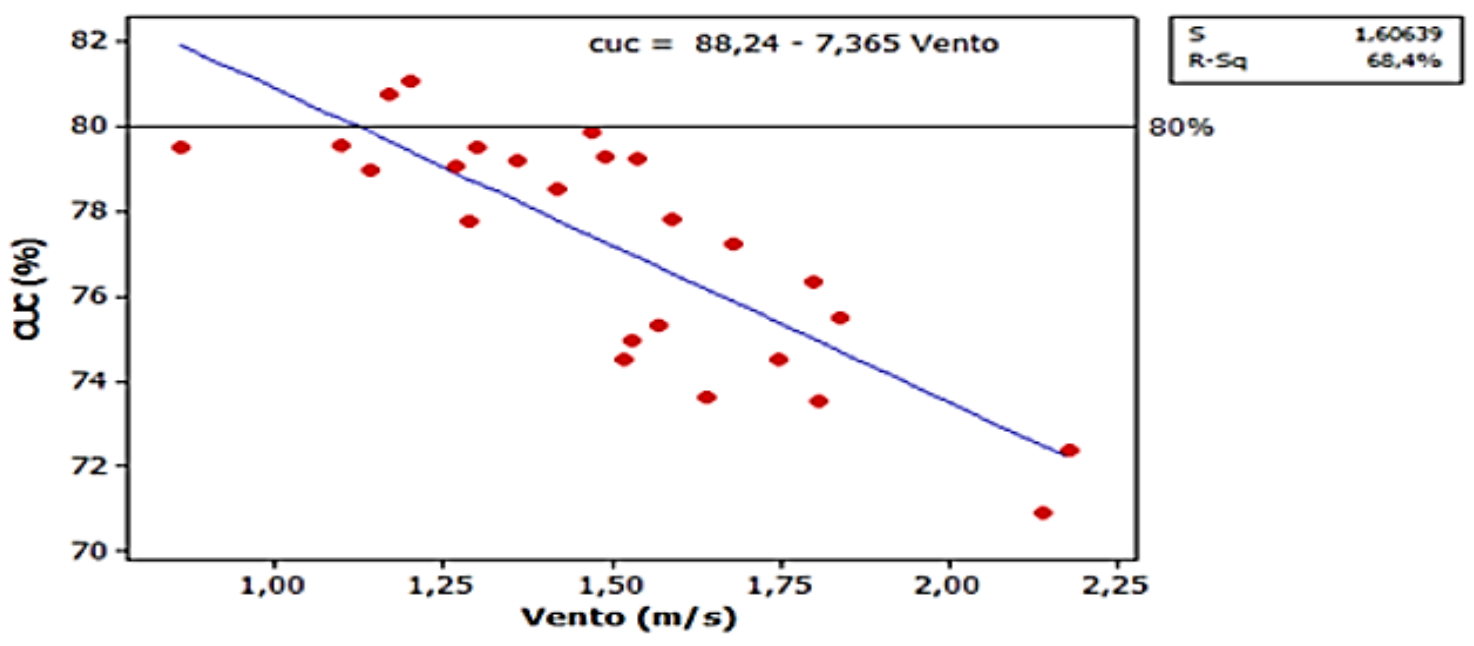

Figura 1. Relação entre CUC e velocidade do vento na fertirrigação. 


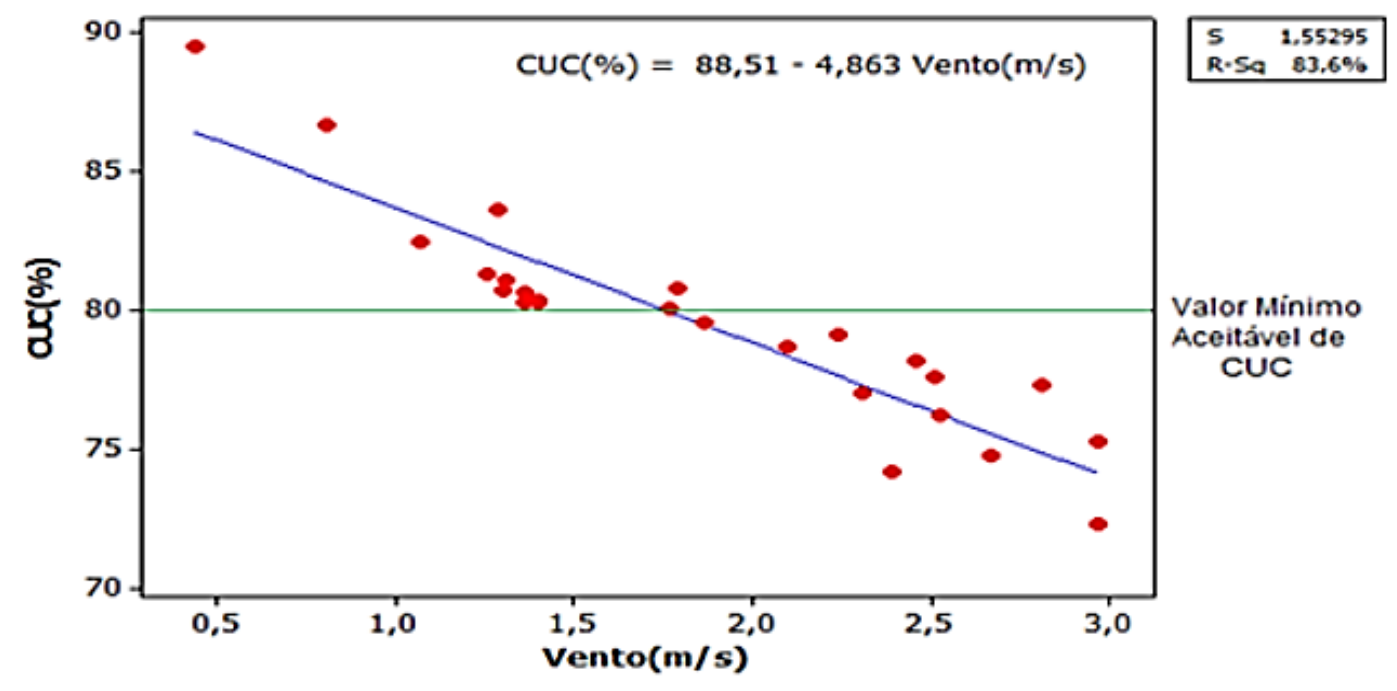

Figura 2. Relação entre CUC e velocidade do vento na irrigação.

relação entre CUC e velocidade do vento semelhante a este estudo, com valores de uniformidade também abaixo do mínimo recomendado de $80 \%$, obtendo um CUC médio de $77,9 \%$, valor próximo ao encontrado neste estudo, cujo valor médio do CUC foi $79,48 \%$.

Azevedo et al. (2000), estudando a influência de fatores climáticos em sistemas por aspersão de alta pressão, encontraram valores de até $50 \%$ de redução do CUC quando do aumento da velocidade do vento, reforçando que assim como foi verificado neste estudo, o vento tem influencia direta na uniformidade de distribuição para a irrigação por aspersão, devendo ser um fator ampla e intensamente monitorado.

Já Playán et al. (2006), verificando a uniformidade de distribuição de água usando modelos balísticoscom uso de três modelos de aspersores, verificaram que, em todos os casos, com o aumento da velocidade do vento, a uniformidade foi afetada sensivelmente, sendo que, para a pressão de serviço de $300 \mathrm{kPa}$, ao testar a regressão linear, encontraram coeficiente de determinação $\mathrm{R}^{2}$ de até $73 \%$, também confirmando a alta influência do vento, como encontrado neste estudo.

Na Figura 3, é apresentado o comportamento do sistema ao longo dos 25 ensaios de irrigação por meio dos gráficos de controle de qualidade de SHEWHART, onde verifica-se que um dos ensaios esteve acima do limite superior de controle (UCL
$=87,52 \%$ ), e nenhum obteve CUC menor que o limite inferior de controle $(\mathrm{LCL}=71,45 \%)$. Os ensaios permaneceram dentro dos limites, sendo que o valor médio foi $79,49 \%$. Exceto o ensaio $8(\mathrm{CUC}=89,45 \%)$, os demais estiveram sob controle, porém com uma grande variação em relação ao valor médio.

Quando avaliado o sistema operando com a fertirrigação, o comportamento dos ensaios pode ser verificado na Figura 4, onde observa-se que um dos ensaios obteve um valor menor que limite inferior de controle (LCL), estimado em 71,28\%, e não houve nenhum ensaio com valor superior ao limite superior de controle (UCL), calculado em $83,01 \%$. A análise do experimento mostra que no caso do valor inferior ao LCL o fator de influência vento foi um dos maiores apresentados durante os ensaios.

Montgomery (2009) afirmou que se um dos pontos aparecerem, ocasionalmente, fora dos limites em gráficos de controle, pode ser resultado de mudanças climáticas, como vento e temperatura, fadiga do operador, alguma variável no equipamento ou, ainda, flutuações na pressão. Cabe salientar que os gráficos foram gerados utilizando-se a sobreposição dos dados dos coletores e que, após 17 ensaios de fertirrigação, ou seja, $17 \mathrm{~h}$ do sistema efetivamente funcionando, um dos emissores apresentou falha e não apresentou rotação, apenas aspergia água, e foi substituído. 


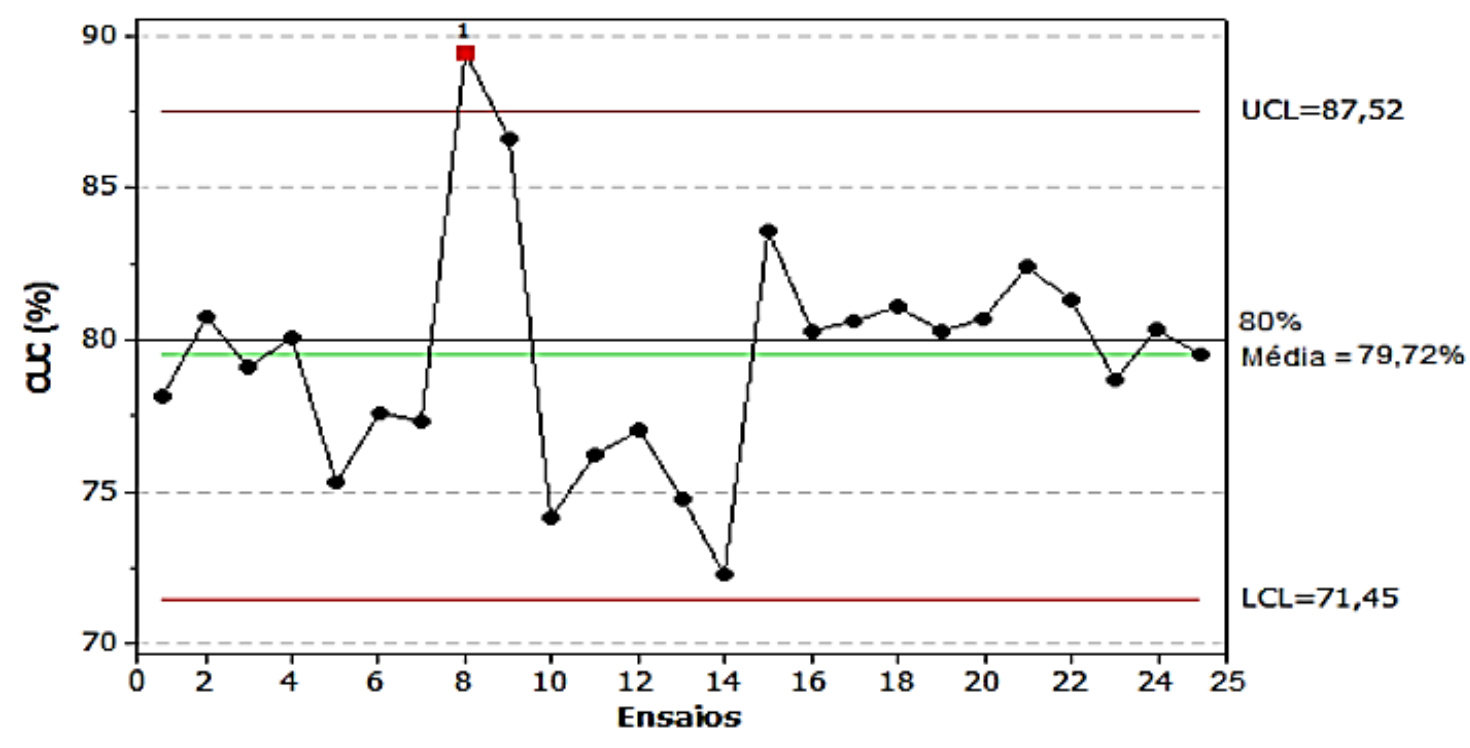

Figura 3. Gráfico de Controle de SHEWHART para a irrigação.

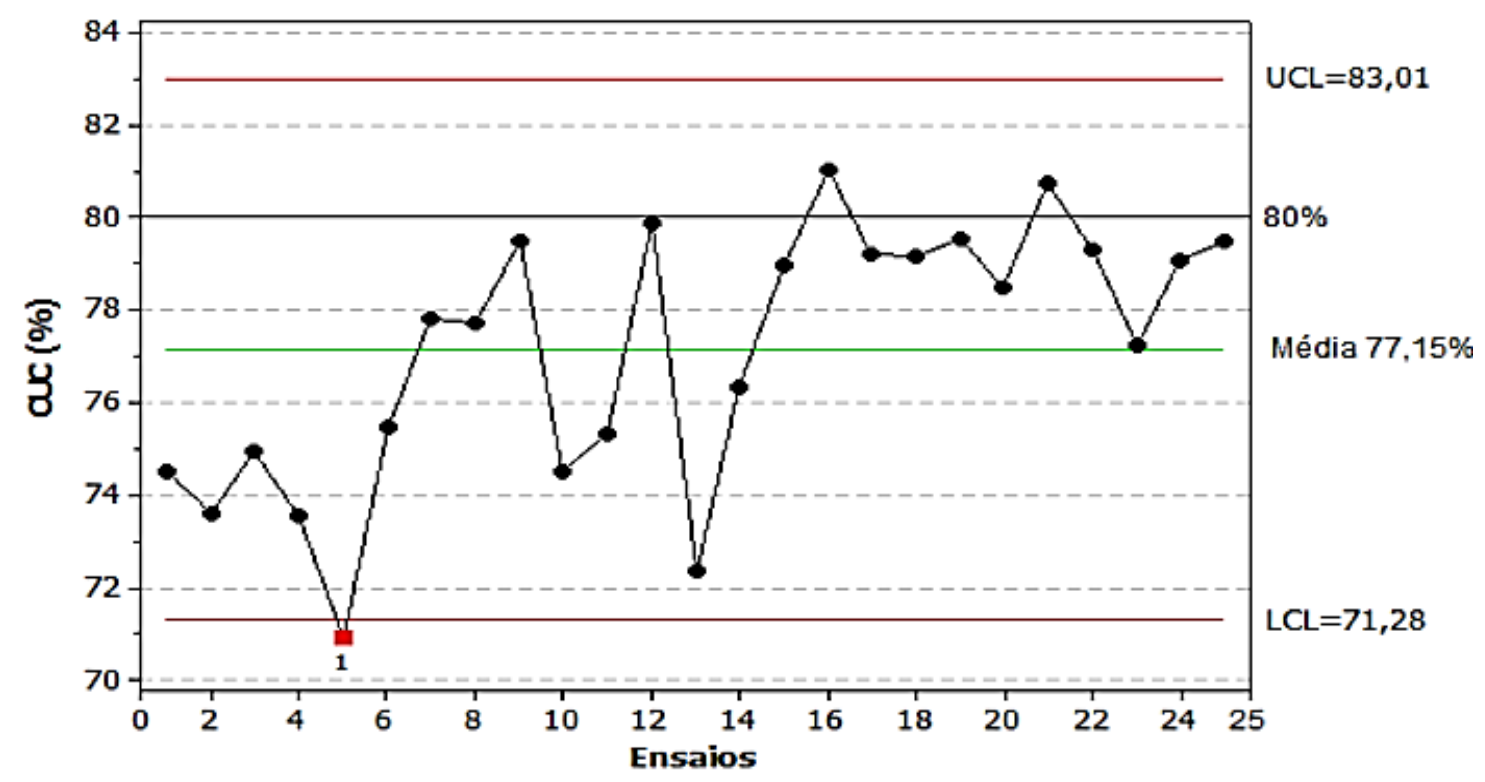

Figura 4. Gráfico de Controle de SHEWHART para a fertirrigação.

No Quadro 3 são apresentados os valores calculados do índice de capacidade do processo (CPL), ao longo dos 25 ensaios. Os valores de CPL encontrados mostram a relação entre o $\mathrm{CPL}$ e a variabilidade, pois quanto maior o valor do CUC, menor foi o desvio padrão. Quanto aos valores de CPL, de acordo com o que Montgomery (2009) afirmou para processos novos, o valor mínimo para o índice de capacidade do processo deve ser 1,60 para que o processo seja considerado aceitável.

Como o desempenho do CUC para a fertirrigação não apresentou normalidade a 5\% de significância, foi necessário realizar uma transformação dos dados, utilizando a transformação BOX-COX, com 
Quadro 3. Valores do índice de capacidade do processo $\left(\mathrm{C}_{\mathrm{PL}}\right)$ para Irrigação e Fertirrigação.

\begin{tabular}{lcc}
\hline & CUC (\%) & $\mathrm{C}_{\mathrm{PL}}$ \\
\hline \multirow{2}{*}{ IRRIGAÇÃO } & $70-75$ & 2,26 \\
& $75-80$ & 2,97 \\
FERTIRRIGAÇÃO & $>80$ & 3,00 \\
& $70-75$ & 1,20 \\
& $75-80$ & 1,66 \\
\hline
\end{tabular}

um valor estimado de lambda em 5.

Hermes etal. (2013)monitorando a uniformidade de um sistema de irrigação por gotejamento com o processamento da mandioca diluída de efluentes verificou que o valor CUC variou entre 85 e $87,5 \%$ , com um índice de capacidade de processo 4,13, e quando os valores variaram entre 87,5 CUC e $90 \%$ , o índice de capacidade de processo foi de 4,19. Quando o valor CUC foi superior a $90 \%$, obteve um índice de capacidade de processo foi de 5,50, reforçando o comportamento encontrado neste estudo, onde o aumento do CUC é proporcional ao aumento do índice de capacidade do processo.

Em seu estudo sobre o índice de capacidade do processo na avaliação da irrigação por aspersão, Justi et al. (2010), concluíram que o índice de capacidade do processo mostrou-se capaz de diagnosticar se a irrigação tem capacidade de se manter sob controle e constante, ou seja, se será capaz de manter níveis aceitáveis de uniformidade. Tal conclusão se alinha aos resultados obtidos neste estudo, onde se verificou a relação entre os maiores valores de CUC com os maiores índices de capacidade do processo encontrados, em concordância com Montgomery (2009), no qual $\mathrm{o}$ autor afirmou que para processos novos, o valor mínimo para o índice de capacidade do processo deve ser 1,60 para que o processo seja considerado aceitável. No caso da irrigação analisada, foi de um sistema novo implantado em campo e pode ser considerado um processo com capacidade aceitável, pois possui índices com valores superiores aos indicados na literatura especializada.

Tal afirmação corrobora ao texto de Rafaelli et al. (2002) em seu trabalho de controle de qualidade e a valiação da capacidade do processo de fabricação de ração monitorando o teor de proteína bruta, teor de água e atividade de ureas e do farelo de soja utilizado como matéria prima, onde os autores citam que quanto maior o valor do índice de capacidade do processo, menor a variabilidade durante o processo.

CHEN et al. (2006), afirmaram após estudar a capacidade do processo para medidas unilaterais que esse índice, utilizando gráficos de controle, pode ser usado não somente para monitorar a estabilidade do processo, mas também para monitorar a qualidade do mesmo, acompanhando índices de especificação e avaliando sua estabilidade, confirmando a viabilidade do uso do controle de qualidade na avaliação de processos.

\section{CONCLUSÃO}

De acordo com o observado nos ensaios, pôdese observar que:

- Apesar de ter grande parte dos ensaios com valor inferior a $80 \%$, o sistema de irrigação estudado apresentou coeficiente de uniformidade de distribuição médio próximo do mínimo aceitável;

- A avaliação de um sistema de irrigação por aspersão utilizando controle estatístico de qualidade por meio de gráficos de controle se apresenta como ferramenta eficiente para o monitoramento da irrigação, pois mostra pontos nos quais o sistema sofreu ação de fatores externos, como no caso a velocidade do vento e a pressão; 
- O uso do controle estatístico de qualidade indica, também, quando a uniformidade oscilou fora dos limites especificados no tempo;

- O uso da capacidade do processo pode diagnosticar se o sistema tem capacidade de se manter dentro de controle e em níveis aceitáveis;

- Houve uma proporção direta entre o aumento da uniformidade de distribuição de água com o aumento do índice de capacidade do processo.

\section{REFERÊNCIAS BIBLIOGRÁFICAS}

AZEVEDO, J.H.; BERNARDO, S.; RAMOS, M.M.; SEDIYAMA, G.C.; CECON, P.R. Influência de fatores climáticos e operacionais sobre a uniformidade de distribuição de água, em um sistema de irrigação por aspersão de alta pressão. Revista Brasileira de Engenharia Agrícola e Ambiental, Campina Grande, v.4, n.2, p.152-158, 2000.

BRENNAN, D. Factors affecting the economic benefits of sprinkler uniformity and their implications for irrigation water use. Irrigation Science. Heidelberg, n.26, p.109 - 119, 2008.

BURT, C. M.; CLEMMENS,A. J.; STRELKOFF, T. S.; SOLOMON, K. H.; BLIESNER, R. D.; HARDY, L. A.; HOWELL, T. A.; EISENHAUER, D. E. Irrigation performancemeasures:Efficien cyand Uniformity. Journalof Irrigation and Drainage. Reston,VA, v.123,n.6, p.423-442, 1997.

CARRIÓN, P.; TARJUELO, J.M.; MONTERO, J. SIRIAS: a simulation model for sprinkler irrigation. Irrigation Science. Heidelberg, n.20, p. $73-84,2001$.

CHEN, K.S.; HUANG, H.L.; HUANG, C.T., Control charts for one-sided capability indices. Quality \& Quantity. n.41,p.413-427, 2006.

CLEMMENS, A.J.; MOLDEN, D.J. Water uses and productivity of irrigation systems. Irrigation Science. Heidelberg, n.25, p.247 - 261, 2007.
FARIA, L.C.; COLOMBO, A.; OLIVEIRA, H.F.E.; PRADO, G.Simulação da uniformidade da irrigação de sistemas convencionais de aspersão operando sob diferentes condições de vento. Engenharia Agrícola. Jaboticabal, n.1, v.29, 2009.

FRIZZONE, J.A.; ANDRADE JÚNIOR, A.S.; SOUZA, J.L.M.; ZOCOLER, J.L. Planejamento da Irrigação: análise de decisão de investimento. 1. ed. Brasília: Embrapa Informação Tecnológica, 2005. v.1. 627p.

FRIZZONE, J.A.; REZENDE, R.; GONÇALVES, A.C.A.; HEBEL JÚNIOR, A. Produtividade do feijoeiro sob diferentes uniformidades de distribuição de água na superfície e na subsuperfície do solo. Engenharia Agrícola, Jaboticabal, v.27, n.2, p.414-425, 2007.

HERMES E.; VILAS BOAS, M.A.; GOMES S.D.; GOMES, B.M; REIS, C.F. Quality control in irrigation and fertigation with cassava processing wastewater into drip system. Journal of Food, Agriculture and Environment. n.2, v.11, p.841$845,2013$.

JUSTI, A.L., VILAS BOAS, M.A.; SAMPAIO, S.C. Índice de capacidade do processo na avaliação da irrigação por aspersão. Engenharia Agrícola. Jaboticabal, n.2, v.30, p.264-270, 2010.

KLAR,

A.E. Critériosparaescolhadométododeirrigação.Irriga, Botucatu, SP, v.5, p.52-82, 2000.

LI, J.; MENG, Y.; LI, B. Field evaluation of fertigation uniformity as affected by injector type and manufacturing variabilty of emitters. Irrigation Science. Heidelberg, v.25,p.117-125,2007.

LI, J.; RAO, M. Field evaluation of crop yield as affected by nonuniformity of sprinklerapplied water and fertilizer. Agricultural Water Management, Amsterdam, n.3, v.76, p.1-13, 2003.

MANTOVANI, E. C.; BERNARDO, S.; PALARETTI, L. F. Irrigação: princípios e 
métodos.3. ed. atual. Viçosa, MG: UFV, 2009. $355 \mathrm{p}$.

MATEOS, L. Assessing whole-field uniformity of stationary sprinkler irrigation systems. Irrigation Science, New York, n.2, v.18, p.73-81, 1998.

MONTGOMERY, D.C. Introduction to statistical quality control. 6. ed. LTC, 513p. 2009.

PLAYÁN, E.; ZAPATA, N.; FACI, J.M.; TOLOSA, D.; LACUEVA, J.L.; PELEGRÍN, J.; SALVADOR, R.; SÁNCHEZ, I.; LAFITA, A. Assessing sprinkler irrigation uniformity using a ballistic simulation model. Agricultural Water Management, Amsterdam, v.84, p.89-100, 2006.

RAFAELLI, D. C.; VILASBOAS, M.A.; URIBEOPAZO, M.A.; Controle de qualidade e avaliação da capacidade do processo de fabricação de ração monitorando-se o teor de proteína bruta, teor de água e atividade de uréase do farelo de soja utilizado como matéria prima. Engenharia Agrícola, Jaboticabal, n.2, v.22, p.229-237, 2002.

SOARES,J.M.; NASCIMENTO,T. Avaliação técnica do sistema de irrigação por aspersão do perímetro irrigado barreiras. Revista Brasileira de Engenharia Agrícola e Ambiental, Campina
Grande, n.2, v.2, p.136-141,1998.

SOLOMON, K.H. Sprinkler Irrigation Uniformity. CATI Research Publications (1990). Disponível em: $\quad$ http://www.atinet.org/newcati/cit/ rese/90/900803/. Acesso em: 2 mar. 2014.

STOUMBOS, Z.G., REYNOLDS JR, M.R., RYAN, T.P., WOODALL, W.H. The state of statistical process control as we proceed into the 21 st century. Journal of the American Statistical Association. n. 95, p.992-998, 2000.

SOUZA, E.A.M., SOUZA, P.C., VILAS BOAS, M.A. Avaliação do desempenho de sistemas de irrigação por aspersão convencional fixo e gotejamento em vila rural. Irriga, Botucatu, n.1, v. 11, p.47-62, 2008.

VEIGA, P.; MENDES, L.; LOURENÇO, L. A retrospective view of statistical quality control research and identification of emerging trends: a bibliometric analysis. Quality \& Quantity, p.120, 2015.

WERKEMA, M.C.C. Ferramentas estatísticas básicas para o gerenciamento de processos. BeloHorizonte, MG: Fundação Cristiano Ottoni, 1995. 404p. 